\title{
Spatial Attention and Saccade Preparation Both Independently Contribute to the Discrimination of Oblique Orientations
}

\author{
Samantha Parker', Andrew Heathcote' ${ }^{2}$, and Matthew Finkbeiner ${ }^{1}$ \\ 'Perception in Action Research Centre and Department of Cognitive Science, Macquarie University, Sydney, Australia \\ 2 Department of Psychology, University of Tasmania, Sandy Bay, Tasmania, Australia
}

\section{KEYWORDS}

spatial attention

saccade

cueing

oblique orientation

evidence accumulation modelling

eye movements
The extent to which the preparation of an eye movement and spatial attention both independently influence performance within the same task has long been debated. In a recent study that combined computational modelling with a dual-task, both saccade preparation and spatial cueing were revealed to separately contribute to the discrimination of targets oriented along the cardinal axis (horizontal and vertical). However, it remains to be seen whether and to what degree the same holds true when different perceptual stimuli are used. In the present study, we combined evidence accumulation modelling with a dual-task paradigm to assess the extent to which both saccade preparation and spatial attention contribute to the discrimination of full contrast targets oriented along the oblique axis (diagonal). The results revealed a separate and quantifiable contribution of both types of orienting to discrimination performance. Comparison of the magnitude of these effects to those obtained for cardinal orientation discrimination revealed the influence of saccade preparation and spatial attention to be six times smaller for oblique orientations. Importantly, the results revealed a separate and quantifiable contribution of both saccade preparation and spatial attention regardless of perceptual stimuli or stimulus contrast.

\section{INTRODUCTION}

Vision researchers have been concerned with the extent to which spatial attention and the preparation of an eye movement are linked or independent for over three decades (Hunt et al. 2019). One particular paradigm that has been used extensively to investigate this relationship is the dual-task. Here, participants are asked to saccade to one element in a display whilst simultaneously detecting or discriminating a perceptual target at the same or a different location. Spatial attention is concurrently manipulated through the use of spatial cues or probability schedules. Studies that do not find any evidence that spatial attention can influence performance at locations away from the saccade goal generally conclude that both forms of orienting are obligatorily linked (Deubel \& Schneider, 1996; Deubel \& Schneider, 2003; Hoffman \& Subramaniam, 1995; Shepherd et al., 1986). Other researchers that report an influence of spatial attention away from the saccade goal typically interpret these results as evidence that each orienting mechanism is independent (Born et al., 2013; Moehler \& Fiehler, 2014, 2015; Montagnini \& Castet, 2007). Only recently has it been possible to unambiguously quantify the relative contribution of these two forms of orienting to dual-task performance (Parker et al., 2020). This is because, to date, the most typical approach has been to use accuracy and response times (RTs) as measures by which to compare the contributions of spatial attention and saccade preparation. However, in a

Corresponding author: Samantha Parker, Department of Cognitive Science, Macquarie University, 16 University Avenue, Macquarie University, NSW 2109, Australia. Email: samantha.parker@mq.edu.au 
traditional analysis of manifest measures such as these, the influence of both spatial attention and saccadic orienting cannot be disentangled from other aspects of dual-task performance. This is a particular problem for the dual-task paradigm, as difficulty complying with the task instructions to saccade to one direction and discriminate a target at the same or a different location requires conditions to be presented across separate blocks. Blocking experimental conditions introduces potential confounds that can limit the conclusions drawn about the operation of orienting specifically. In a previous study, we overcame this limitation by combining evidence accumulation modelling with a saccadic dual-task for the first time (Parker et al., 2020). The quality of evidence accumulation was used as a common currency by which to compare the influence of saccade preparation and spatial attention. The results revealed an independent and measurable influence of both saccade preparation and spatial attention during the discrimination of vertical and horizontal targets. While the size of the saccade congruency effect remained relatively stable across two separate dual-task paradigms, the magnitude of the spatial cueing effect varied by cue type. The influence of spatial attention was greater when directed by a peripheral, rather than central, cue.

One question that remains unanswered is the extent to which these orienting effects are specific to the perceptual stimuli and task employed. To date, the magnitudes of these effects have only been assessed during the discrimination of stimuli oriented along the cardinal axes and where contrast has been individually adjusted. Therefore, the goal of the present study was to quantify the contribution of saccade preparation and spatial attention to the discrimination of obliquely oriented, full contrast stimuli. In doing so, our aims were twofold. First, we sought to use the computational method to compare the magnitude of the spatial attention and saccade preparation effects in a distinct perceptual task. Second, we sought to confirm that the influence of spatial attention and saccade preparation on perception was independent of target orientation and contrast (Castet et al., 2006; Montagnini \& Castet, 2007). In doing so, our results shed new light on the circumstances and degree to which orienting contributes to perceptual performance.

\section{Contribution of Orienting Across Perceptual Tasks}

The findings from previous dual-task studies have, to date, been difficult to reconcile. While some authors reported an independent influence of spatial attention away from the saccade goal (Born et al., 2013; Castet et al., 2006; Moehler \& Fiehler, 2014, 2015; Montagnini \& Castet, 2007), others found evidence to suggest the relationship is obligatory (Deubel \& Schneider, 1996; Deubel \& Schneider, 2003; Hoffman \& Subramaniam, 1995; Shepherd et al., 1986). Part of the issue is that the experimental design of these studies varies greatly. One factor that often differs between studies is the perceptual task employed. Some authors, for example, require participants to detect a target letter or numeral amongst distractors (Deubel, 2008; Deubel \& Schneider, 1996; Deubel \& Schneider, 2003; Dore-Mazars et al., 2004; Hoffman \& Subramaniam, 1995), whilst others require participants to discriminate the orientation (Castet et al., 2006; Moehler \& Fiehler, 2018; Montagnini \& Castet, 2007) or the offset of a target (Born et al., 2012; Born et al., 2013). These tasks differ in many ways. For example, letter discrimination requires high spatial acuity and is generally performed better at fovea (Anstis, 1974). Orientation discrimination, on the other hand, requires less spatial resolution and can be performed well in peripheral vision (Pardiso \& Carney, 1988). Given these differences, it is reasonable to suspect that the relative effects of saccade preparation and spatial attention may also differ by task.

Despite this, there has been no way to measure and compare the contribution of orienting across these distinct experimental designs. In a typical dual-task, accuracy and RTs are analysed separately. The limitation of analysing accuracy and RT separately is that it is not clear which is the best way to combine these measures into a single metric by which to compare task difficulty across conditions (Wagenmakers et al., 2007). For example, the speed-accuracy trade-off phenomenon (Fitts, 1966; Ratcliff \& Rouder, 1998) refers to decision behaviour where cautious responding is associated with slower but more accurate choices, and less cautious responding is associated with faster but more error prone responding, even when task difficulty remains the same. This phenomenon makes it difficult to draw conclusions about performance across experimental tasks from a separate analysis of accuracy and RT. For example, if, a participant exhibited the above pattern of responding across a letter and orientation discrimination task, respectively, it would not be apparent from an analysis of accuracy and RT alone which task was more difficult or, indeed, if there was any difference in the difficulty of the tasks at all.

Evidence accumulation modelling, on the other hand, allows the contribution of each orienting mechanism to be quantified and compared in a common currency. It does this by combining accuracy and the distribution of RTs for correct and error responses to estimate the parameters of a model that can separate the effects of response caution from task difficulty (Donkin et al., 2009, 2011). While accumulation models differ with respect to their architecture, they all share the same three basic parameters; threshold, drift rate, and nondecision time. Thresholds measure the amount of evidence necessary to trigger a response, and can therefore account for speed-accuracy trade-off behaviour. Drift rate is a measure of the deterministic component of a decision that captures both the quality and speed of information processing (Lewandowsky \& Oberauer, 2018; Wagenmakers et al., 2007). Nondecision time is the duration of all other non-decision activities, such as the time taken to encode the stimulus and make a motor response. By assessing how these parameters vary as a function of experimental manipulation, we can extract a direct, common, and theoretically interpretable measure of orienting and drift rate, that is separate from differences in response caution. It is these properties of parameter estimates that allow the influence of orienting to be compared across blocked conditions and distinct tasks/stimuli.

Previously, we used this approach to measure the contribution of saccade preparation and spatial attention to the discrimination of cardinal orientations. In using an accumulation model, we were able to not only quantify the effect of both types of orienting across blocked 
saccade conditions, but also to compare the relative influence of spatial attention when directed by a peripheral versus a central cue (Parker et al., 2020). Specifically, when a peripheral cue was employed, the magnitude of the saccade preparation effect (1.16) was revealed to be similar in size to that of the peripheral cue (0.88). Here, we used the same method to measure the influence of orienting to oblique orientation discrimination, and for the first time, assessed whether, and to what extent, these effects vary by perceptual task. In doing so, we also sought to confirm that these influences were independent of target contrast and orientation.

\section{Contrast}

It is well established that both spatial attention and saccade preparation alike modulate the detection and perception of contrast (Cameron et al., 2002; Carrasco et al., 2004; Ling \& Carrasco, 2006; Lu \& Dosher, 2000; Pestilli \& Carrasco, 2005; Rolfs \& Carrasco, 2012). For example, one widely reported finding is that spatial attention increases contrast sensitivity at attended locations and impairs it at unattended locations (Pestilli \& Carrasco, 2005). Similar research has even suggested that spatial attention modulates the subjective appearance of contrast, such that attention enhances the perceived contrast of a given stimulus (Carrasco et al., 2004). Complementary research into the perceptual consequences of preparing an eye movement has shown that the mere preparation of a saccade can also modulate the perceived contrast of a target at its upcoming location. Specifically, saccade preparation has been shown to enhance a participant's subjective perception of contrast at the upcoming saccade goal, with participants perceiving stimuli at this location to be of a higher contrast than stimuli at other locations (Rolfs \& Carrasco, 2012).

These findings suggest that spatial attention and saccade preparation alike can modulate both contrast sensitivity and the subjective perception of contrast. This is significant because a number of previous studies reporting an influence of spatial attention at locations independent of the saccade goal manipulated the contrast of discrimination stimuli (Castet et al., 2006; Montagnini \& Castet, 2007). For example, Parker et al. (2020), in the computational study outlined above, individually adjusted the contrast of target and distractors for each participant. This was done to ensure that performance on an orientation discrimination task was not at ceiling. An unintended consequence, however, was that it is not clear to what extent these orienting effects depend upon manipulations in contrast. That is, as both spatial attention and the preparation of an eye movement modulate contrast sensitivity, it is possible that these independent orienting effects do not generalize to stimuli presented at full contrast. In order to address this question, in the current study, we measured the magnitude of the saccade congruency and spatial cueing effect for full contrast stimuli.

\section{Oblique Versus Cardinal Orientations}

Discrimination performance is also known to vary by orientation. Stimuli along the cardinal axes are discriminated faster and more accurately than stimuli along the oblique axes (Appelle, 1972; Furmanski \& Engel, 2000; Heeley et al., 1997). This anisotropy has also been found to interact with attentional load (Bloem \& Ling, 2017) and the preparation of an eye movement (Lee \& Lee, 2008). Greater attentional load is found to impair the detection of oblique orientations to a greater extent than cardinal (Bloem \& Ling, 2017), while saccade preparation has been reported to have the opposite effect on discrimination performance. That is, there is a decreased sensitivity to cardinal orientations at the goal of an upcoming eye movement about $100 \mathrm{~ms}$ before saccade onset (Lee \& Lee, 2008). Again, these findings are important because the magnitude of each type of orienting has, to date, only been measured across one specific perceptual task, namely, cardinal orientation discrimination (Parker et al., 2020). Given that research suggests not only that the discrimination of oblique orientations is more difficult, but that each orientation may be uniquely influenced by attention and saccade preparation, it is possible that the contributions of spatial attention and saccade preparation may vary by orientation task. Therefore, we sought to assess to what extent both spatial attention and saccade preparation contributed to oblique orientation discrimination, and whether the magnitude of these effects differed when compared to cardinal orientation discrimination.

\section{The Current Study}

The aim of the current paper was to measure the relative influence of saccade preparation and spatial attention to an oblique orientation discrimination task. With this in mind, we used a similar experimental design and computational modelling approach to that employed previously (see Parker et al., 2020, Experiment 2). Saccade preparation was manipulated by having participants prepare and execute eye movements towards one of three different placeholders (left, right, or above fixation), separated across blocks. An auditory tone signalled the onset of an eye movement. The discrimination target, an obliquely angled Gabor patch surrounded by a vertical Gabor patch of the same spatial frequency, could then appear in any of the three locations. Spatial attention was directed through the use of a peripheral cue which could appear at any of the three placeholder positions. The inclusion of three placeholder positions meant that we could vary the angle at which the target appeared from both the saccade goal and spatial cue. Some studies have found the benefit of preparing an eye movement to be spatially distributed around the saccade goal (Castet et al., 2006), while more recent research suggests that these effects are largely confined to the saccade goal (Szinte et al., 2019; Wollenberg et al., 2018). Consistent with this latter approach, Parker et al. (2020) found no evidence to suggest that spatial attention or saccade preparation effects were spatially distributed. In the current study, we examined whether this is true for obliquely oriented stimuli. If the saccade preparation effect is spatially distributed, then performance should be better when the target appears $90^{\circ}$ from the saccade goal, relative to $180^{\circ}$. Similar predictions follow for spatial attention if these effects are spatially distributed.

Importantly, the participant's task was to report the angle of tilt (left or right) of the target, compared to the upper half of the surrounding vertical Gabor. To ensure performance was off ceiling rather than manipulate contrast, the angle of tilt was adjusted to an $82 \%$ threshold in a separate staircase procedure that set the angle of the target throughout the main task. 
The relative contribution of each orienting mechanism was assessed by fitting the linear ballistic accumulator (LBA; Brown \& Heathcote, 2008) to data from the dual-task. In order to account for any differences in response caution that can exist across blocked conditions, we allowed the threshold parameter of the LBA model, the criterion level of information that must be accumulated from the environment in order to trigger a choice or response, to vary by saccade direction (left, right, or up). Our primary dependent measure was drift rate, the rate at which evidence accumulates towards the threshold. Estimates of this parameter are able to capture both the quality and quantity of information accumulation. Specifically, by taking the difference in drift rate between an accumulator for the correct response (i.e., the rate at which evidence accumulates for the response that matches the stimulus or the "true" accumulator) and the incorrect response (i.e., the rate at which evidence accumulates for the response that mismatches the stimulus or the "false" accumulator), we can quantify the quality of information in the system. That is, larger differences in drift rate between the true and false accumulator represent a higher quality of information accumulating from the stimulus (Boag et al., 2019). The drift rate parameter allowed us to quantify the relative contributions of saccade preparation and covert spatial attention to performance and separate this influence from other factors in the dual-task design that may influence performance (Lewandowsky \& Oberauer, 2018). Therefore, drift rate can be used as a measure by which to compar e the quantitative effects of orienting across distinct studies. In quantifying and comparing these effects across tasks, we expected that, consistent with our previous study (Parker et al., 2020), there would be a separable and measurable influence of both saccade preparation and s patial cueing to oblique orientation discrimination.

\section{METHOD}

\section{Participants}

Twenty-four participants (6 males) from Macquarie University took part in the experiment in return for course credit. All experimental procedures were approved by the local ethics committee and all participants gave informed consent before participation. Participant age ranged from 19 to 29 years $(M=20.13, S D=2.06)$. All participants had normal or corrected to normal vision. Eight participants were replaced either because they did not perform reliably better than chance $(N=6)$ or they did not make the correct eye movement on a sufficient number of trials $(N=2)$. Power simulations (Brysbaert \& Stevens, 2018) using standard estimates taken from a previous study (saccade congruency $b=1.01$, cue validity $b=0.81$, Parker et al., 2020 ) confirmed that there was sufficient power (80\%) for an a level of .05 with a sample size of 24 ..

\section{Stimuli}

The experiment was run and all stimuli were generated using PsychoPy (Peirce et al., 2019). All stimuli were presented white against a grey background. Discrimination targets were diagonally oriented Gabor patches $\left(1.23^{\circ}\right.$ in diameter) generated by modulating a sine wave ( 1.6 cycles per degree) with a Gaussian envelope $(S D=$ $\left.0.35^{\circ}\right)$. All targets were surrounded by a vertically oriented Gabor patch $\left(2.06^{\circ}\right.$ diameter $)$ with the same spatial frequency as the target. Distractors were vertically oriented Gabor patches $\left(2.06^{\circ}\right.$ diameter). Possible target location was indicated by three placeholder squares $\left(3.04^{\circ} \times 3.04^{\circ}\right)$ positioned $2.73^{\circ}$ to the left, right, and above the fixation (see Figure 1). Target location was cued by one of the placeholders turning pink.

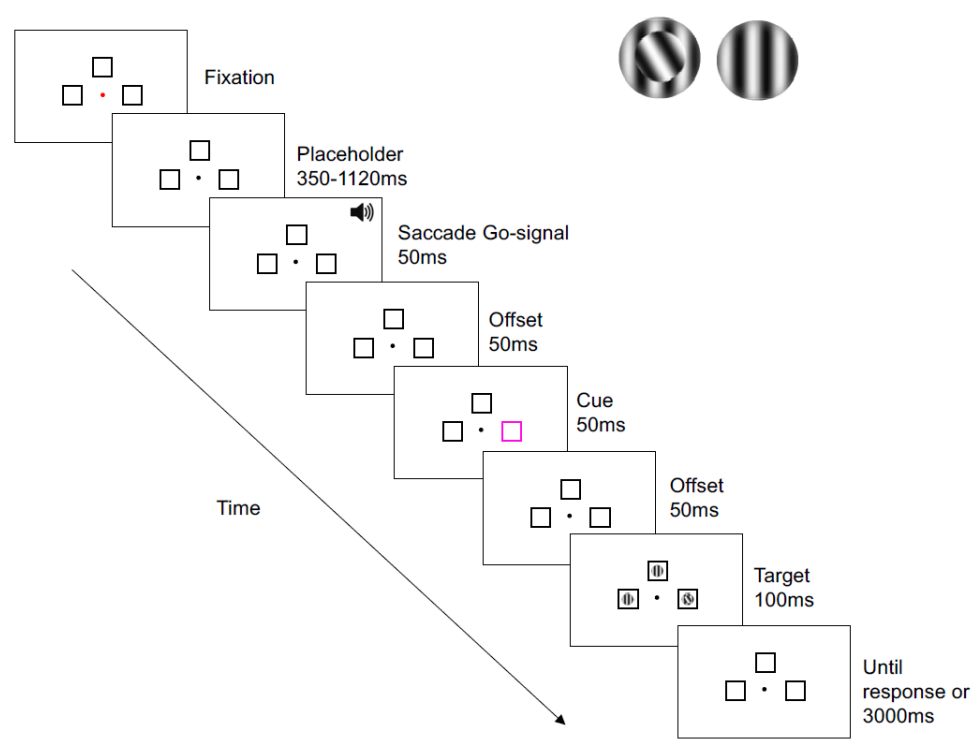

FIGURE 1.

Trial schematic of a valid trial. Inset is example of target and distractor stimulus, respectively. Note that stimuli were presented as white against a grey background. 


\section{Design}

The experiment followed a $3 \times 3$ factorial design where saccade congruency had three levels (congruent, incongruent-180 ${ }^{\circ}$, and incongruent $-90^{\circ}$ ) and cue validity had three levels (valid, invalid- $180^{\circ}$, and invalid- $\left.90^{\circ}\right)$. Trials in which the target appeared at the placeholder above fixation could not occur in the incongruent- $180^{\circ}$ or invalid- 180 ${ }^{\circ}$ condition. Therefore, in order to maintain an equal number of trials in each condition, we excluded these trials from our analysis. In a separate analysis, we confirmed that this decision did not significantly alter the pattern of results ${ }^{1}$. Incongruent- $180^{\circ}$ trials were trials where the saccade goal was directly opposite the target $\left(180^{\circ}\right)$ and incongruent- $90^{\circ}$ trials were where the saccade goal appeared at an angle of 90 ${ }^{\circ}$ from the target. The spatial cue appeared at the same locations with respect to the target in the invalid- $180^{\circ}$ and invalid- $90^{\circ}$ conditions. Participants completed 108 practice trials, followed by 144 trials of a QUEST staircase procedure (Watson \& Pelli, 1983), where two interleaved staircases adjusted the angle of the target relative to its vertical background to an $82 \%$ accurate threshold. The angle ranged from $\pm 10-45^{\circ}$ from vertical, adjusted for each individual participant $(M=$ $\left.33^{\circ}, S D=12^{\circ}\right)$. The outcome of this staircase procedure set the angle that was maintained throughout the main task. The main experiment consisted of 252 trials per saccade direction for a total of 756 trials. Participants received a break screen after every 80 trials and at the start of a new eye movement direction, with the experiment taking approximately two hours to complete. The eye tracker was calibrated using a 9-point calibration procedure at the start of every block and as necessary throughout the task.

\section{Procedure}

Participants were informed at the outset of each block that they must simultaneously complete both the saccade and orientation discrimination task. For the saccade task, participants were told that upon the onset of an auditory tone, they must saccade to the left, right, or upward of the placeholder box. Saccade direction (left, right, or up) was separated into three blocks and the order of the blocks was counterbalanced across every 6th participant. The participants' task was an oblique orientation decision in which they were required to indicate on a keyboard whether a target was tilted to the right or left. Participants were told to make their tilt judgement with reference to the top half of the stimulus.

Participants sat in a darkened room before an LED monitor (1920 $\times 1080 \mathrm{p}, 120 \mathrm{~Hz}$ ). A chin and forehead rest positioned on the edge of the desk, at a distance of $85 \mathrm{~cm}$ from the monitor, stabilized the participants' head throughout the experiment. An Eyelink 1000 Desktop Mount eye tracker (SR Research) monitored the position of the participants' right eye throughout the experiment $(500 \mathrm{~Hz})$. The trial commenced when participants pressed the space bar while fixating on the small red circle in the centre of the screen. The first frame displayed the three white placeholder squares. The duration of this frame was drawn from an exponential function (minimum of $350 \mathrm{~ms}$ ). If the duration exceeded the maximum of $1120 \mathrm{~ms}$, the trial was terminated $(\sim 10 \%$ of all trials). This was done to ensure the participants had a flat hazard rate with respect to the beginning of the trial and the onset of the eye movement signal (Ghose \& Maunsell, 2002). On the remaining trials, an auditory tone played for $50 \mathrm{~ms}$. The tone signalled to participants to make an eye movement. After a further $50 \mathrm{~ms}$, one of three white placeholders was cued by drawing the outline in pink for $50 \mathrm{~ms}$. Each placeholder location was equally likely to be cued and the cue was nonpredictive about the location of the target. After $50 \mathrm{~ms}$, the target was displayed for $100 \mathrm{~ms}$. The target could appear in any of the placeholder positions and was, therefore, validly cued on one third of all trials. Distractors accompanied the onset of the target in the two remaining placeholder squares. The participants had $3 \mathrm{~s}$ to respond by pressing the left or right arrow key to indicate whether the target was leaning to the left or right or the trial timed out. The participants received two types of visual feedback. "Correct" or "Incorrect" indicated whether the correct orientation discrimination decision had been made, while the colour of the feedback (green or red) indicated whether the correct eye movement has been made. If no saccade was detected within 750 ms of tone onset, the participants received the feedback "too slow."

\section{Gaze Data Analysis}

Eye movements were monitored both online and offline. Online monitoring of eye movements ensured that participants maintained central fixation until the onset of the auditory go-signal. If a saccade was detected in any direction more than $1.45^{\circ}$ from fixation before the tone, the trial was immediately terminated and recycled. A correct saccade was defined as one that landed within the instructed placeholder square. An offline algorithm was used to determine the onset of the saccades. At each time point, raw eye position data was smoothed with a Gaussian function. The average velocity of five neighbouring time points was then used to compute a smoothed average eye velocity. Saccades were detected when eye velocity exceeded the median velocity by more than 5 SDs for at least $8 \mathrm{~ms}$ (Engbert \& Kliegl, 2003). Trials containing blinks, incorrect saccades ( $9 \%$ of all trials), or eye movements initiated before the offset of the target were eliminated $(9.4 \%$ of all trials). Only trials in which the eyes were at fixation while the target was onscreen were compared. As is typical in the dual-task literature, any trial where saccades were initiated more than $450 \mathrm{~ms}$ after target offset were eliminated ( $0.4 \%$ of all trials, Born et al., 2013; Castet et al., 2006; Moehler \& Fiehler, 2014, 2015, 2018). This meant that a total of $18.8 \%$ of all trials were excluded from subsequent analysis.

\section{RESULTS}

As a first step, we conducted an analysis of accuracy, correct RT, and saccade latency as a function of saccade congruency and cue validity. Generalised linear mixed modelling for accuracy data (GLMM) and linear mixed effects modelling (LMM) for correct RT and saccade latency data was implemented in $R$ using the lme4 software package (Bates et al., 2015). The reliability of each effect of interest was evaluated using the incremental modelling approach in which goodness of fit statistics (AIC, BIC, and Log likelihood values; Akaike, 1974; Schwarz, 1978) were used to determine which of our models provided the best fit 


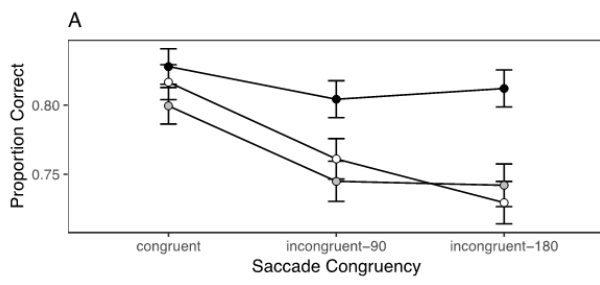

Cue Validity

- valid

invalid-90

- invalid-180

B

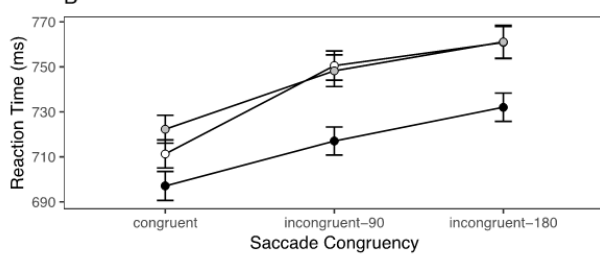

Cue Validity

- valid

invalid-90

- invalid-180

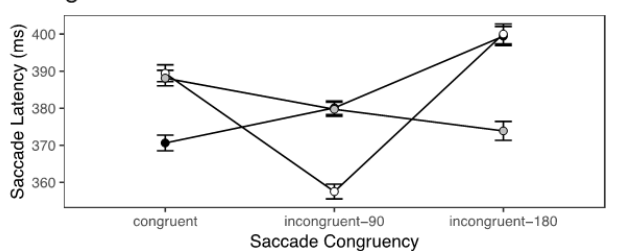

Cue Validity

- valid

invalid-90

FIGURE 2.

Saccade congruency and cue validity effect in accuracy (Panel A), reaction time (in milliseconds, Panel B), and saccade latency (in milliseconds, Panel C). Error bars represent within subject SEMs.

for our data. This procedure involved comparing a model that included the effect of interest with one that excluded the effect of interest. Only effects that significantly improved the fit of the model were included in the analysis. Trials in which RT exceeded 3 SDs of the participants mean were excluded from the analysis (2.1\% of trials). As it is typical in dual-task studies, to analyse data as a function of time between target offset and saccade onset, but not central to the aim of our study, we included an analysis by absolute time in the Supplementary Materials (Born et al., 2013; Deubel, 2008).

\section{Manifest Analysis}

\section{ACCURACY}

The incremental modelling procedure revealed the model of best fit to include a main effect of saccade congruency, $\chi^{2}(2)=28.20, p<.001$, and cue validity, $\chi^{2}(2)=28.73, p<.001$. Accuracy was better when the discrimination target and saccade goal were congruent $(M=0.80, S D=$ $0.13)$ relative to when they were at a $90^{\circ},(M=0.76, S D=0.14 ; b=-0.29$, $S E=0.07, z=-4.26, p<.001$,$) or a 180^{\circ}$ angle, $(M=0.75, S D=0.13 ; b=$ $-0.34, S E=0.07, z=-5.06, \mathrm{p}<.001)$ from each other. A follow-up $t$-test confirmed there was no significant difference between incongruent- $90^{\circ}$ or $180^{\circ}$ trials, $t(23)=0.38, p=.71$. Similarly, accuracy was higher when the target was preceded by a valid cue $(M=0.80, S D=0.13)$ relative to a cue located $90^{\circ}(M=0.76, S D=0.13 ; b=-0.30, S E=0.07, z=-4.29, p<$ $.001)$ or $180^{\circ}$ from the target $(M=0.75, S D=0.14 ; b=-0.34, S E=0.07$, $z=-4.95, p<.001$, see Figure 2, Panel A). Again, there was no evidence to suggest that accuracy differed for invalid $-90^{\circ}$ or $180^{\circ}$ trials, $t(23)=$ $0.81, p=0.71$.

\section{REACTION TIME}

An LMM with correct RT as the dependent measure revealed the model of best fit to include a main effect of saccade congruency, $\chi^{2}(2)=$ $66.63, p<.001$, and cue validity $\chi^{2}(1)=38.18, p<.001$. Button press RTs were faster when the discrimination target appeared at the goal of an upcoming eye movement $(M=722 \mathrm{~ms}, S D=111)$ relative to appearing at a location at $90^{\circ}(M=742, S D=110 ; b=28.62, S E=5.03, t=5.70)$ or $180^{\circ}$ from the saccade goal $(M=764, S D=128 ; b=41.35, S E=5.06, t$ $=8.17)$. There was no evidence to suggest that $\mathrm{RT}$ differed whether the incongruent location was $90^{\circ}$ or $180^{\circ}$ from the target, $t(23)=-1.54, p$ $=0.27$. Reaction times were also faster when the discrimination target was preceded by a valid $(M=724 \mathrm{~ms}, S D=109)$ relative to an invalid cue at an angle of $90^{\circ}(M=751, S D=122 ; b=25.07, S E=5.05, t=4.96)$ and $180^{\circ}(M=754, S D=120 ; b=28.58, S E=5.06, t=5.65)$. Again, a t-test did not reveal any evidence to suggest that RTs significantly differed whether the placeholder $90^{\circ}$ or $180^{\circ}$ from the target was cued, $t(23)=-0.82, p=421$.

\section{SACCADE LATENCY}

The incremental modelling procedure revealed the model of best fit to include a main effect of saccade congruency, $\chi^{2}(2)=113.00, p<.001$, and an interaction between saccade congruency and cue validity, $\chi^{2}$ $(4)=213.94, p<.001$. This interaction is best understood as a conflict effect between saccade goal and cue location. That is, eye movements were slower when the spatial cue appeared at a location incongruent to the saccade goal, relative to when it appeared at a congruent location. We therefore recoded trials as a function of this spatial cue-saccade goal congruency. Three FDR adjusted pairwise $t$-tests revealed latencies to be significantly faster towards the spatial cue $(M=367, S D=$ 38) compared to opposite it $(M=393, S D=40 ; t(23)=-8.43, p<.001)$ or at a $90^{\circ}$ angle from it $(M=386, S D=41), t(23)=-5.86, p<.001$. A $t$-test confirmed that latencies were also slower when a saccade was required to the placeholder opposite the cue relative to at a $90^{\circ}$ angle, $t(23)=2.27, p=.03$

\section{Linear Ballistic Accumulator Analysis}

\section{MODEL SPECIFICATION}

We fit the linear ballistic accumulator (LBA) to each participant's data. The LBA models have one accumulator for each response, each with potentially different parameter values. Therefore, there was one accumulator for left leaning targets, and one for right leaning targets. Each accumulator had the following parameters: start point noise, representing the range of evidence values in each accumulator at the beginning of the decision, which was assumed to follow a uniform distribution with a range of 0 to $A \geq 0$; drift rate, the rate at which evidence towards a decision is accumulated, which was assumed to follow a normal distribution with a mean of $v$ and a $S D$ of $s_{v} \geq 0$; response threshold, the amount of evidence necessary to trigger a decision, 
denoted by $b$ (note that the results here are reported in terms of the difference between the top of the start point distribution and the response threshold, $B=b-A \geq 0$ ); and non-decision time, $T_{\mathrm{er}} \geq 0$, which we assumed to be the same for both accumulators (Donkin et al., 2011; Heathcote et al., 2002)

In order to describe the LBA parameterisation, we defined a response accumulator factor " $\mathrm{R}$ ", with levels that corresponded to the left and right accumulators, and an accumulator correspondence factor, "C", which denoted the "true" (matching) and "false" (mismatching) accumulator for each stimulus. For example, if the target was offset towards the left, then the left accumulator is the "true" accumulator and the right is the "false" accumulator. The difference between the drift rates for the true and false accumulators is a measure of the quality of information accumulation, with larger differences indicative of a greater quality of information in the system (Boag et al., 2019). Therefore, difference between the true and false accumulators was the primary dependent measure by which to compare the relative contributions of orienting. Response bias was modelled by allowing $B$ to vary with the response factor. Above-chance performance requires a higher evidence accumulation rate for the true than false accumulator, and this was modelled by allowing $v$ to vary with the correspondence factor.

We allowed threshold to vary as a function of saccade instruction (up, left, or right), as these conditions were blocked. Drift rate was allowed to vary by saccade congruency and cue validity. The sv parameter was allowed to vary with the correspondence factor (Heathcote et al., 2002; Heathcote \& Love, 2012), and $s_{\mathrm{v}}$ for the mismatching parameter was fixed at 1 to make the model identifiable (Donkin et al., 2009). A single value for all conditions of $A$ and $T_{\mathrm{er}}$ were estimated.

\section{MODEL FIT}

Separate model fits to each participant's data were obtained using maximum likelihood estimation. We used the optimization (parameter search) method to obtain fits, fitting models with fewer parameters, and then used the best fitting parameters for the simple models as starting points for the best fitting parameters of more complicated models (Donkin et al., 2011). Graphical summaries, included in the Supplementary Materials, confirmed that our selected models were able to capture the major trends in the data (Heathcote et al., 2002).

\section{PARAMETER ESTIMATES}

Thresholds. First, threshold estimates $(B)$ were assessed using a LMM, which included an effect of blocked saccade instruction (left, right, and up) and response (right or left tilt). The LMM of best fit was revealed to include a main effect of saccade instruction, $\chi^{2}(2)=11.91$, $p=.002$. Thresholds were higher for trials that required a saccade to the placeholder above fixation (2.16) relative to trials that required an eye movement towards the left $(1.96 ; b=-0.20, S E=0.07, t=-3.10)$ or right placeholders $(1.97 ; b=-0.19, S E=0.07, t=-2.93)$.

Drift rate. Our main dependent measure of interest was the quality of information accumulation, quantified as the difference between the true and false drift rate. A difference score was computed between each accumulator (true minus false) for every subject and every condition.
This difference score was then submitted to a LMM to determine if saccade congruency and cue validity modulated the quality of information accumulation. The LMM revealed the model of best fit to include a main effect of saccade congruency, $\chi^{2}(2)=16.82, p<.001$, and cue validity, $\chi^{2}(2)=18.05, p<.001$.

We followed this analysis by quantifying the magnitude of these effects. As the manifest analysis revealed no evidence to suggest that performance differed whether the saccade goal or spatial cue appeared at an angle of $90^{\circ}$ or $180^{\circ}$ from the target, we collapsed "incongruent" and "invalid" trials to create a single condition. To quantify the size of the saccade congruency effect, we took the difference between the true and false drift rate on trials where the saccade goal was congruent with the target vs. trials in which the saccade goal was incongruent. The same comparison was made for valid relative to invalid trials. The results revealed the effect of saccade congruency $(0.20)$ to be of a similar magnitude to the spatial cueing effect (0.14, see Figure 3$)$. There was no evidence to suggest that the magnitude of these effects significantly differed, $t(23)=1.45, p=.16$.

\section{Saccade Latency Modeling}

There was a significant conflict effect in saccade latencies such that eye movements were initiated fastest when the spatial cue and saccade goal coincided, slower when they were at an angle of $90^{\circ}$, and slowest when they were directly opposite each other. As an analysis of latencies alone does not allow us to characterise the nature of this effect, we followed up our manifest analysis by fitting an LBA model to each participant's saccade latency data. In particular, we examined whether this conflict effect was best explained by modulations in the drift rate parameter, which measures the speed with which a response is made and quantifies task performance, or the nondecision time parameter, a measure of all nondecision processes involved in the task. If the conflict effect was best explained by the drift rate component of the model, this would suggest that saccade preparation and spatial attention are yoked, and that this yoking influences the perception of the target. If, instead,

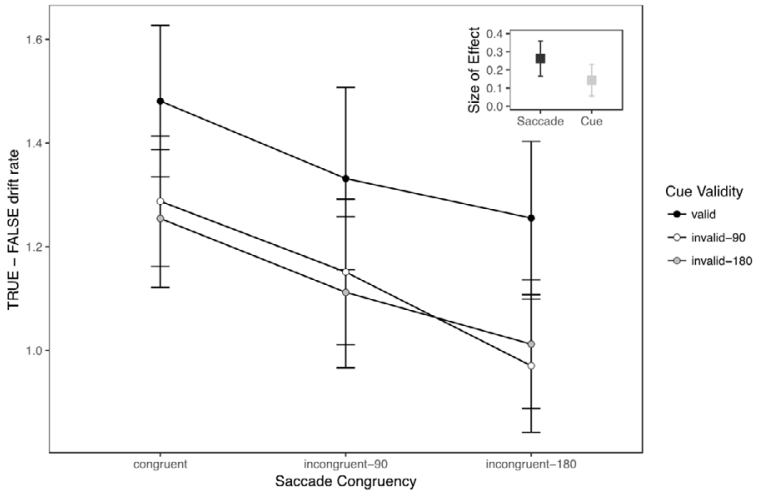

FIGURE 3.

Difference between true and false drift rate for saccade congruency and cue validity. Main graph shows the saccade congruency and cue validity effects in the quality of information accumulation. Inset shows the magnitude of these effects. Error bars are within-subject SEMs. 
the conflict was best explained by the nondecision component of the model, this would be good evidence that this conflict occurs outside the decision stage of the dual-task, perhaps during the motor execution of an eye movement.

\section{Model Specifications}

The model only had a single accumulator corresponding to correct eye movements, as these were the only trials included in the analysis. Nondecision time $\left(T_{0}\right)$ and drift rate $(v)$ were allowed to vary by whether the saccade goal and spatial cue coincided (congruent), were at an angle of $90^{\circ}$ (incongruent- $90^{\circ}$ ), or were directly opposite each other (incongruent- $180^{\circ}$ ). A single value was estimated for all conditions of $\mathrm{A}$ and $\mathrm{B}$, and we fixed $s_{\mathrm{v}}=1$ to make the model identifiable.

\section{Model Fit}

The maximum likelihood model estimation software used above does not allow for fitting a single accumulator, so we used the more flexible DMC Software (Heathcote et al., 2018), which carries out estimation in a Bayesian manner. We outline the priors and sampling procedure in the Supplementary Materials. Sampling occurred in two steps. First, sampling was carried out separately for individual participants. The results of this procedure were then used as starting points for the full hierarchical model, whose results are reported below. Model fit was good and cumulative distribution functions are presented in the Supplementary Materials.

\section{Parameter Estimates}

Parameter estimates are reported as posterior medians with $95 \%$ credible intervals given in square brackets. The effects of cue-congruency are tested using Bayesian $p$-values to test the difference in parameters between conditions (Klauer, 2010) by tabulating differences between pairs of parameters. The $p$ values correspond to the proportion of differences where congruent was greater than incongruent- $90^{\circ}$ or incongruent- $180^{\circ}$. Hence, small p-values support better performance in the congruent condition. Nondecision time was revealed to be $0.017 \mathrm{~s}$ faster when the cue and saccade goal were congruent relative to incongruent- $180^{\circ}(0.193 \mathrm{~s}[0.190,0.196]$ vs. $0.210 \mathrm{~s}[0.207,0.213], p<.001)$. Nondecision time was also $0.017 \mathrm{~s}$ faster when the cue and saccade goal was congruent compared to incongruent- $90^{\circ}(0.193 \mathrm{~s}[0.190,0.196]$ vs. $0.210 \mathrm{~s}[0.207,0.213], p<.001)$. There was no evidence to suggest nondecision time differed whether the spatial cue and saccade goal were at an angle of $180^{\circ}$ or $90^{\circ}$ from each other. Nor was there any evidence to suggest that the drift rate parameter varied by cue congruency.

\section{DISCUSSION}

The aim of the current study was to quantify the relative contributions of saccade preparation and spatial attention to the discrimination of oblique orientations. Specifically, we sought to measure the magnitude of the saccade congruency and spatial cueing effect to an oblique orientation discrimination task that employed full contrast stimuli. In using an evidence accumulation model, we were able to establish that there is a unique and quantifiable influence of saccade preparation and spatial attention to oblique orientation discrimination for full contrast stimuli.

\section{Independent and Measurable Influence of Saccade Preparation and Spatial Attention}

Consistent with our previous study (Parker et al., 2020), there was an influence of both saccade congruency and spatial cueing on performance. The saccade congruency effect was apparent in manifest measures, where accuracy was higher and response times faster when the saccade goal and discrimination target coincided relative to when they did not (Deubel, 2008; Hoffman \& Subramaniam, 1995; Kowler et al., 1995; Shepherd et al., 1986). There was no apparent difference in performance when a saccade was directed away from the target and towards a placeholder positioned $90^{\circ}$ or $180^{\circ}$ from the target. Accumulator modelling confirmed that saccade congruency modulated the quality of information accumulation. There was a higher quality of signal accruing from the discrimination target when it appeared at the same location as an upcoming eye movement, relative to an incongruent location. Critically, there was also an effect of spatial cueing. Regardless of the direction an eye movement was prepared, accuracy was better and RTs were faster when the target was preceded by a valid compared to an invalid cue (Born et al., 2013; Deubel, 2008; Moehler \& Fiehler, 2014, 2015; Montagnini \& Castet, 2007). We found no evidence to suggest that performance differed when the cue appeared 90 ${ }^{\circ}$ or $180^{\circ}$ from the target. Spatial cueing was similarly confirmed to modulate the quality of information accumulation, with higher quality signal accumulating from the target when it was preceded by a valid rather than invalid spatial cue (Carrasco \& McElree, 2001; Smith \& Ratcliff, 2009; Smith et al., 2004).

We then quantified the magnitude of these effects in the threshold and drift rate parameters of the LBA model. The results revealed the threshold parameter to vary by saccade instruction (left, right, or up). Thresholds were significantly higher for the block in which participants were required to saccade towards the placeholder positioned directly above fixation compared to blocks which required rightward or leftward saccades. While unexpected, the effect of saccade instruction on thresholds may reflect biological and perceptual differences between vertical and horizontal saccades. While originally assumed to be similar (Smit et al., 1987), research has now established not only that there are anatomical differences between the generation and execution of vertical and horizontal saccades (Bonnet et al., 2013; Leigh \& Zee, 2015), but that vertical saccades are subject to more interference from distractors than horizontal eye movements (Laidlaw \& Kingstone, 2010). It is possible that participants require a greater amount of information to trigger responses on upward saccade trials because there is a larger amount of interference when the cue and target appear along the horizontal axis. Importantly, unlike an analysis of accuracy and RT, the computational modelling approach allowed us to account for these differences in threshold. By allowing the threshold parameter to vary by saccade direction, these differences can be isolated and we can extract an unambiguous measure of orienting. 
In quantifying the influence of saccade congruency and cue validity in drift rate our results revealed an independent and measurable influence of both saccade preparation and spatial attention to the discrimination of oblique orientations. The magnitude of the saccade congruency (0.20) and spatial cueing (0.14) effects were revealed to be similar. Indeed, consistent with our previous study, follow-up analysis revealed no evidence to suggest that they differed significantly in magnitude from each other. Critically however the results of the present study did replicate our previous findings. This was true even though the perceptual task was more difficult and the stimuli were presented at full-contrast.

There was however a conflict effect between cue location and saccade goal evident in the saccade latencies. That is, participants were slower to move their eyes in a direction away from the spatial cue relative to towards the cue. At first glance, this finding may be interpreted as evidence that spatial attention and saccade programming are obligatorily linked. Slower latencies on cue-incongruent trials may be because participants first plan an eye movement in the direction of the cue, then must reprogram and execute an eye movement towards the final saccade goal. However, an alternative explanation is that this conflict effect, rather than reflecting a perceptual coupling between orienting mechanisms, is best explained as a type of saccadic bottleneck. That is, when the spatial cue and saccade goal are in conflict, there is a conflict in the saccade execution stage that must be resolved before an eye movement can be programmed. Therefore, this conflict influences the motor components of the response rather than the perceptual aspects of the decision. The LBA modelling of saccade latency data confirmed that the conflict effect was best explained by the nondecision time parameter of the model. A finding that suggests this latency effect is best explained by a bottleneck in the saccade execution stage. This result further suggests that spatial attention is not always obligatorily tied to the goal of an upcoming eye movement.

\section{Comparison of Magnitudes Across Oblique and Cardinal Orientation Discrimination}

A significant advantage of computational modelling is that it allows the influence of each type of orienting to be measured in a common currency that can be used to make meaningful comparisons across different dual-task designs. This is possible because in fitting an evidence accumulation model, factors that may influence performance on a dual-task can be isolated and accounted for. In doing so, a quality measure of how orienting contributes to the deterministic component of a decision can be extracted. Using this approach, we have previously shown that the contribution of spatial attention to task performance varies by cue type. That is, when spatial attention is directed with a peripheral cue, there is a much larger influence of spatial attention on perception than when it is directed by a centrally presented arrow. In the current study, we extended this approach to examine how the contributions of spatial attention and saccade preparation vary across distinct perceptual tasks and stimuli for the first time. Specifically, we were able to compare the effects obtained in the current study with obliquely oriented stimuli to those previously reported with cardinally oriented targets (Parker et al., 2020).

Importantly, despite finding an independent and quantifiable influence of both spatial attention and saccade preparation, the relative sizes of these effects were approximately six times (84\%) smaller than those previously reported (Parker et al., 2020). This was true despite the manipulation of saccade congruency and spatial cueing being identical to the current paradigm. Therefore, we attribute this magnitude effect to the use of different perceptual stimuli, and specifically to the difficulty of discriminating oblique orientations in the current study relative to cardinal orientations in the previous study. However, difficulty does not refer to overall accuracy performance, but rather the computational differences between discriminating oblique and cardinal orientations. Individual performance on the oblique task was more variable than that reported for vertical and horizontal stimuli. This trial-to-trial variability may have contributed to smaller overall effects of saccade congruency and spatial cueing. Some of this variability likely stems from the modification of the target stimulus. In contrast to previous studies, the target stimulus in the present study was composed of a vertical Gabor $\left(2.06^{\circ}\right.$ in diameter) superimposed by a smaller oblique Gabor $\left(1.23^{\circ}\right)$. This target stimulus may have influenced variability in two ways. First, the superimposed Gabor, which contained the oblique information necessary for a decision, was smaller than the targets in the previous study. Second, our discrimination target may have been difficult to detect due to orientation-surround suppression. Studies have shown that the discrimination of a stimulus can be affected by the orientation of the surrounding stimulus, particularly when the orientations are similar in angle (Petrov \& McKee, 2006; Polat \& Sagi, 1993). It is possible that by superimposing our target stimulus on a reference vertical Gabor, there was an increased amount of suppression from the surrounding Gabor making oblique orientations even more difficult to judge. Other research has found exogenously oriented spatial attention and saccade preparation to modulate orientation information distinctly. Fernandez et al. (2019) reported that while exogenous spatial attention enhanced the gain of orientation information, the preparation of an eye movement also narrowed orientation tuning.

Regardless of the overall difference in magnitude between these two studies, the relative contribution of spatial attention and saccade preparation remained similar across both studies. Again, we found no evidence to suggest that the saccade congruency and spatial cueing effect differed significantly in magnitude when a peripheral cue was employed. However, modelling enabled us to detect a small but unique and measurable impact of spatial attention away from the saccade goal. Our results not only confirm that there are independent effects of spatial attention and saccade congruency to perception, but, for the first time, allow the extent of these effects to be compared across unique discrimination tasks. Results suggest that while the magnitude of each orienting mechanisms varies by perceptual task, there is still a measurable and unique influence of spatial attention independent from the saccade goal (for further discussion, see Parker et al., 2020). 


\section{Conclusion}

The current study establishes two important findings. First, it confirms that there is a unique influence of both saccade preparation and spatial attention to dual-task performance, replicating our original findings. Second, it establishes a method by which to compare the relative influence of orienting across distinct perceptual tasks. A central issue in the study of spatial attention and saccadic programming is the lack of consistency in paradigms used to investigate this relationship (Born et al., 2014). This has led to a large number of studies, with some suggesting that spatial attention and saccade preparation are linked, and others arguing to the contrary. Importantly, until now, there has been no way to reconcile these results. In the dual-task literature alone, which represents only one type of paradigm used to investigate this relationship, the typical dual-task design varies along a number of different dimensions. For example, how saccades are directed and blocked, how spatial attention is manipulated and what type of perceptual task is employed can all differ between tasks. Our results are significant not only because they confirm that spatial attention is not always obligatorily tied to the goal of a saccade, but also because they establish a new way to measure the influence of orienting across different paradigms. Using this method, we established that when experimental design factors are accounted for, there is still a unique and measurable influence of both spatial attention and saccade preparation to the discrimination of oblique orientations at full contrast.

\section{FOOTNOTES}

${ }^{1}$ The linear mixed effects modelling (LMM) and generalized linear mixed modelling (GLMM) procedure revealed the same pattern of results as that reported here, when "target up" trials were included.

\section{ACKNOWLEDGEMENTS}

This work was supported in part by an Australian Research Council Grant to A.H. (Grant No. DP160101891) and an Australian Government Research Training Program Scholarship to S.P.

\section{REFERENCES}

Akaike, H. (1974). A new look at the statistical model identification. IEEE Transactions on Automatic Control, 19, 716-723. doi: 10.1109/ TAC.1974.1100705 الس الس

Anstis, S. M. (1974). A chart demonstrating variations in acuity with retinal position. Vision Research, 14, 589-592. doi: 10.1016/00426989(74)90049-2 سلس

Appelle, S. (1972). Perception and discrimination as a function of stimulus orientation: The Oblique Effect in man and animals. Psychological Bulletin, 78, 266-278. doi: 10.1037/h0033117 لسلسلس

Bates, D., Maechler, M., Bolker, B., \& Walker, S. (2015). Fitting linear mixed-effects models using lme4. Journal of Statistical Software, 67, 1-48. doi: 10.18637/jss.v067.i01 الس

Bloem, I. M., \& Ling, S. (2017). Attentional modulation interacts with orientation anisotropies in contrast perception. Journal of Vision, 17, 1-14. doi: 10.1167/17.11.6 السلسلس

Boag, R. J., Strickland, L., Loft, S., \& Heathcote, A. (2019). Strategic at- tention and decision control support prospective memory in a complex dual-task environment. Cognition, 191, 1-24. doi: 10.1016/j. cognition.2019.05.011

Bonnet, C., Hanuska, J., Rusz, J., Rivaud-Pechoux, S., Sieger, T., Majerova, V., . . Ruzicka, E. (2013). Horizontal and vertical eye movement metrics: What is important? Clinical Neurophysiology,

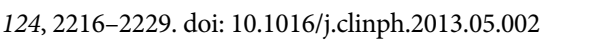

Born, S., Ansorge, U., \& Kerzel, D. (2012). Feature-based effects in the coupling between attention and saccades. Journal of Vision, 12, 1-17. doi: 10.1167/12.11.27 سلس

Born, S., Ansorge, U., \& Kerzel, D. (2013). Predictability of spatial and non-spatial target properties improves perception in the presaccadic interval. Vision Research, 91, 93-101. doi: 10.1016/j.vis-

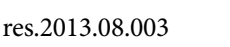

Born, S., Mottet, I., \& Kerzel, D. (2014). Presaccadic perceptual facilitation effects depend on saccade execution: Evidence from the stop-

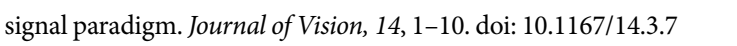

Brown, S. D., \& Heathcote, A. (2008). The simplest complete model of choice response time: linear ballistic accumulation. Cognitive Psychology, 57, 153-178. doi: 10.1016/j.cogpsych.2007.12.002 السلمالسلاسل

Brysbaert, M., \& Stevens, M. (2018). Power analysis and effect size in mixed effects models: A tutorial. Journal of Cognition, 1, 1-20. doi:10.5334/joc.10 السلبل

Cameron, E. L., Tai, J. C., \& Carrasco, M. (2002). Covert attention affects the psychometric function of contrast sensitivity. Vision Research, 42, 949-967. doi: 10.1016/S0042-6989(02)00039-1 1 سلسلس

Carrasco, M., Ling, S., \& Read, S. (2004). Attention alters appearance. Nature Neuroscience, 7, 308-313. doi: 10.1038/nn1194 السلسليلس

Carrasco, M., \& McElree, B. (2001). Covert attention accelerates the rate of visual information processing. Proceedings of the National Academy of Sciences, 98, 5363-5367. doi: 10.1073/pnas.081074098 الس السلسلا

Castet, E., Jeanjean, S., Montagnini, A., Laugier, D., \& Masson, G. S. (2006). Dynamics of attentional deployment during saccadic programming. Journal of Vision, 6, 196-212. doi: 10.1167/6.3.2

Deubel, H. (2008). The time course of presaccadic attention shifts. Psychological Research, 72, 630-640. doi: 10.1007/s00426-008-0165-3 المالسلسلس Deubel, H., \& Schneider, W. X. (1996). Saccade target selection and object recognition: Evidence for a common attentional mechanism. Vision Research, 36, 1827-1837. doi: 10.1016/0042-6989(95)00294-4 سلس

Deubel, H., \& Schneider, W. X. (2003). Delayed saccades, but not delayed manual aiming movements, require visual attention shifts. Annals of the New York Academy of Sciences, 1004, 289-296. doi: 10.1196/annals.1303.026 الس السلس

Donkin, C., Brown, S. D., \& Heathcote, A. (2009). The overconstraint of response time models: Rethinking the scaling problem. Psychonomic Bulletin and Review, 16, 1129-1135. doi: 10.3758/PBR.16.6.1129 الس الس الس

Donkin, C., Brown, S., \& Heathcote, A. (2011). Drawing conclusions from choice response time models: A tutorial using the linear ballistic accumulator. Journal of Mathematical Psychology, 55, 140-151. doi: 10.1016/j.jmp.2010.10.001 للسلسلس

Dore-Mazars, K., Pouget, P., \& Beauvillain, C. (2004). Attentional selection during preparation of eye movements. Psychological Research, 
69, 67-76. doi: 10.1007/s00426-003-0166-1 1 السلس

Engbert, R., \& Kliegl, R. (2003). Microsaccades uncover the orientation of covert attention. Vision Research, 43, 1035-1045. doi: 10.1016/ s0042-6989(03)00084-1 سلس

Fernandez, A., Li, H. H., \& Carrasco, M. (2019). How exogenous spatial attention affects visual representation. Journal of Vision, 19, 4. doi: 10.1167/19.11.4 سلس

Fitts, P. M. (1966). Cognitive aspects of information processing: III. Set for speed versus accuracy. Journal of Experimental Psychology, 71, 849-857. doi: 10.1037/h0023232

Furmanski, C. S., \& Engel, S. A. (2000). An oblique effect in human primary visual cortex. Nature Neuroscience, 3, 535-536. doi:

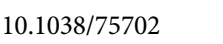

Ghose, G. M., \& Maunsell, J. H. R. (2002). Attentional modulation in visual cortex depends on task timing. Nature, 419, 616-620. doi: 10.1038/nature01057 السلس

Heathcote, A., Brown, S., \& Mewhort, D. J. K. (2002). Quantile maximum likelihood estimation of response time distributions. Psychonomic Bulletin Review, 9, 349-401. doi: 10.3758/BF03196299 السلس

Heathcote, A., Lin, Y.-S., Reynolds, A., Strickland, L., Gretton, M., \& Matzke, D. (2018). Dynamic models of choice. Behavior Research Methods, 51, 961-985. doi: 10.3758/s13428-018-1067-y المالسلسلئل

Heathcote, A., \& Love, J. (2012). Linear deterministic accumulator models of simple choice. Frontiers in Psychology, 3, 1-19. doi: 10.3389/fpsyg.2012.00292 الميلس

Heeley, D. W., Buchanan-Smith, H. M., Cromwell, J. A., \& Wright, J. S. (1997). The oblique effect in orientation acuity. Vision Research, 37, 235-242. doi: 10.1016/S0042-6989(96)00097-1 الهالسلس

Hoffman, J. E., \& Subramaniam, B. (1995). The role of visual attention in saccadic eye movements. Perception and Psychophysics, 57, 787-795. doi: 10.3758/BF03206794

Hunt, A. R., Reuther, J., Hilchey, M. D., \& Klein, R. M. (2019). The relationship between spatial attention and eye movements. Current Topics in Behavioral Neurosciences, 41, 255-278. doi: 10.1007/7854_2019_95 السلسلسلة

Klauer, K. C. (2010). Hierarchical multinomial processing tree models: A latent-trait approach. Psychometrika, 75, 70-98. doi: 10.1007/

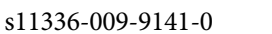

Kowler, E., Anderson, E., Dosher, B., \& Blaser, E. (1995). The role of attention in the programming of saccades. Vision Research, 35, 1897-1916. doi: 10.1016/0042-6989(94)00279-U سلس

Laidlaw, K. E., \& Kingstone, A. (2010). The time course of vertical, horizontal and oblique saccade trajectories: Evidence for greater distractor interference during vertical saccades. Vision Research, 50, 829-837. doi: 10.1016/j.visres.2010.02.009 الفلسلس

Lee, J., \& Lee, C. (2008). Changes in orientation discrimination at the time of saccadic eye movements. Vision Research, 48, 2213-2223. doi: 10.1016/j.visres.2008.06.014 سلس

Leigh, R. J., \& Zee, D. S. (2015). The neurology of eye movements (5 ed.). Oxford: Oxford University Press.

Lewandowsky, S., \& Oberauer, K. (2018). Computational modeling in cognition and cognitive neuroscience. In E.-J. Wagenmakers (Ed.),
Stevens' handbook of experimental psychology and cognitive neuroscience (Vol. 5, pp. 1-35). John Wiley \& Sons Inc.

Ling, S., \& Carrasco, M. (2006). Sustained and transient covert attention enhance the signal via different contrast response functions. Vision Research, 46, 1210-1220. doi: 10.1016/j.visres.2005.05.008 البلسلس

Lu, Z.-L., \& Dosher, B. A. (2000). Spatial attention: Different mechanisms for central and peripheral temporal precues? Journal of Experimental Psychology: Human Perception and Performance, 26, 1534-1548. doi: 10.1037/0096-1523.26.5.1534 المالسلس

Moehler, T., \& Fiehler, K. (2014). Effects of spatial congruency on saccade and visual discrimination performance in a dual-task paradigm. Vision Research, 105, 100-111. doi: 10.1016/j.visres.2014.10.001 س山لा

Moehler, T., \& Fiehler, K. (2015). The influence of spatial congruency and movement preparation time on saccade curvature in simultaneous and sequential dual-tasks. Vision Research, 116, 25-35. doi: 10.1016/j.visres.2015.09.006

Moehler, T., \& Fiehler, K. (2018). Effects of central and peripheral cueing on perceptual and saccade performance. Vision Research, 143, 26-33. doi: 10.1016/j.visres.2017.12.002 السلسلسا

Montagnini, A., \& Castet, E. (2007). Spatiotemporal dynamics of visual attention during saccade preparation: Independence and coupling between attention and movement planning. Journal of Vision, 7, 1-16. doi: 10.1167/7.14.8 السلسلس

Pardiso, M. A., \& Carney, T. (1988). Orientation discrimination as a function of stimulus eccentricity and size: Nasal/temporal retinal asymmetry. Vision Resarch, 28, 867-874. doi: 10.1016/00426989(88)90096-X पلس سلس

Parker, S., Heathcote, A., \& Finkbeiner, M. (2020). Using evidence accumulation modelling to quantify the relative contributions of spatial attention and saccade preparation in perceptual tasks. Journal of Experimental Psychology: Human Perception and Performance, 46, 416-433. doi: 10.1037/xhp0000723 سلس

Peirce, J., Gray, J. R., Simpson, S., MacAskill, M., Hochenberger, R., Sogo, H., . . Lindelov, J. K. (2019). PsychoPy2: Experiments in behavior made easy. Behavioral Research Methods, 51, 195-203. doi: 10.3758/s13428-018-01193-y السلسلس

Pestilli, F., \& Carrasco, M. (2005). Attention enhances contrast sensitivity at cued and impairs it ancued locations. Vision Research, 45,

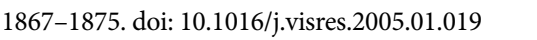

Petrov, Y., \& McKee, S. P. (2006). The effect of spatial configuration on surround suppression of contrast sensitivity. Journal of Vision, 6,

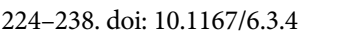

Polat, U., \& Sagi, D. (1993). Lateral interaction between spatial channels: Suppression and facilitation revealed by lateral masking experiments. Vision Research, 33, 993-999. doi: 10.1016/0042-

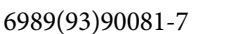

Ratcliff, R., \& Rouder, J. N. (1998). Modeling response times for two-choice decisions. Psychological Science, 9, 347-356. doi:

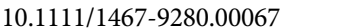

Rolfs, M., \& Carrasco, M. (2012). Rapid simultaneous enhancement of visual sensitivity and perceived contrast during saccade prepa- 
ration. Journal of Neuroscience, 32, 13744-13752a. doi: 10.1523/ JNEUROSCI.2676-12.2012 السلسلس

Schwarz, G. E. (1978). Estimating the dimensions of a model. Annals of

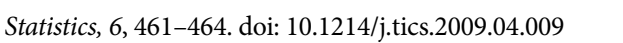

Shepherd, M., Findlay, J. M., \& Hockey, R. J. (1986). The relationship between eye movements and spatial attention. The Quarterly Journal of Experimental Psychology Section A, 38, 475-491. doi: 10.1080/14640748608401609 المالسلبل

Smit, A. C., Van Gisbergen, J. A. M., \& Cools, A. R. (1987). A parametric analysis of human saccades in different experimental paradigms. Vision

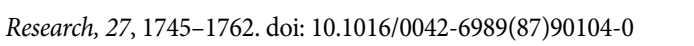

Smith, P. L., \& Ratcliff, R. (2009). An integrated theory of attention and decision making in visual signal detection. Psychological Review,

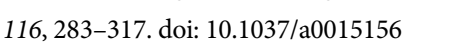

Smith, P. L., Ratcliff, R., \& Wolfgang, B. J. (2004). Attention orienting and the time course of perceptual decisions: Response time distributions with masked and unmasked displays. Vision Research, 44, 1297-1320. doi: 10.1016/j.visres.2004.01.002 المالسلسلس
Szinte, M., Puntiroli, M., \& Deubel, H. (2019). The spread of presaccadic attention depends on the spatial configuration of the visual scene. Scientific Reports, 9, 1-11. doi: 10.1038/s41598-019-50541-1 المالسلسل Wagenmakers, E.-J., van der Maas, H. L. J., \& Grasman, R. P. P. P. (2007). An EZ-diffusion model for response time and accuracy. Psychonomic Bulletin and Review, 14, 3-22. doi: 10.3758/BF03194023 الس السلس

Watson, A. B., \& Pelli, D. G. (1983). QUEST: A Bayesian adaptive psychometric method. Perception and Psychophysics, 33, 113-120. doi: 10.3758/BF03202828 السلس

Wollenberg, L., Deubel, H., \& Szinte, M. (2018). Visual attention is not deployed at the endpoint of averaging saccades. PLoS Biology, 16, 1-23. doi: 10.1371/journal.pbio.2006548 الس 


\section{SUPPLEMENTARY MATERIALS}

\section{Manifest Analysis Including Absolute Time Bin}

Trials were divided into four separate time bins. This led to disproportionate bin sizes. Trials where the saccade occurred within $100 \mathrm{~ms}$ of target offset comprised 62\%, trials with saccade latencies between 101 and $200 \mathrm{~ms}$ of target offset comprised $23 \%$, trials with saccade latencies between 201 and $300 \mathrm{~ms}$ comprised 11\%, while trials with saccade latencies greater than $300 \mathrm{~ms}$ comprised $4 \%$. We included absolute time bin as a factor in our analysis.

\section{ACCURACY}

The incremental modelling procedure revealed that the inclusion of saccade congruency, $\chi^{2}(2)=28.20, p<.001$, and cue validity, $\chi^{2}(2)=$ $28.73, p<.001$, improved the fit of the model. Accuracy was best when the discrimination goal and saccade target were congruent $(M=0.80$, $S D=0.13)$ relative to when they appeared at a $90^{\circ}(M=0.76, S D=0.14$; $b=-0.29, S E=0.07, z=-4.26, p<.001)$ and $180^{\circ}$ angle $(M=0.75$, $S D=0.13 ; b=-0.34, S E=0.07, z=-5.06, p<.001)$ from each other. Similarly, accuracy was also best when the cue was valid $(M=0.80, S D$ $=0.13)$ compared to when it was invalid at an angle of both $90^{\circ}(M=$ $0.76, S D=0.13 ; b=-0.30, S E=0.07, z=-4.29, p<.001)$ and $180^{\circ}(M$ $=0.75, S D=0.14 ; b=-0.34, S E=0.07, z=-4.95, p<.001$, see Figure A1, Panel A).

\section{REACTION TIME}

A linear mixed effects modelling (LMM) with response time (RT) as the dependent measure revealed the model of best fit to include a main effect of saccade congruency, $\chi^{2}(2)=66.63, p<.001$, and cue validity, $\chi^{2}(2)=38.18, p<.001$. Button press response times were faster when the saccade goal and discrimination target were congruent $(M=$ $722 \mathrm{~ms}, S D=111)$ relative to at an angle of $90^{\circ}(M=742 \mathrm{~ms}, S D=110$; $b=28.62, S E=5.03, t=5.70)$ or $180^{\circ}(M=764 \mathrm{~ms}, S D=128 ; b=41.35$, $S E=5.06, t=8.17)$. Response times were also faster when the cue was valid $(M=724 \mathrm{~ms}, S D=109)$ compared to when cue appeared $90^{\circ}(M$ $=751 \mathrm{~ms}, S D=122 ; b=25.07, S E=5.05, t=4.96)$ or $180^{\circ}$ from the target $(M=754 \mathrm{~ms}, S D=120 ; b=28.58, S E=5.06, t=5.65)$.

\section{SACCADE LATENCY}

A LMM revealed the model of best fit to include a main effect of saccade congruency, $\chi^{2}(2)=113.00, p<.001$, absolute time bin, $\chi^{2}(3)$ $=16030.37, p<.001$, and an interaction between saccade congruency and cue validity, $\chi^{2}(4)=121.33, p<.001$. The main effect of time bin was unsurprising given that saccade latency defined the trials in each bin. The interaction between saccade congruency and cue validity is best understood as a conflict effect, where eye movements are slower to initiate away from the trial relative to towards.
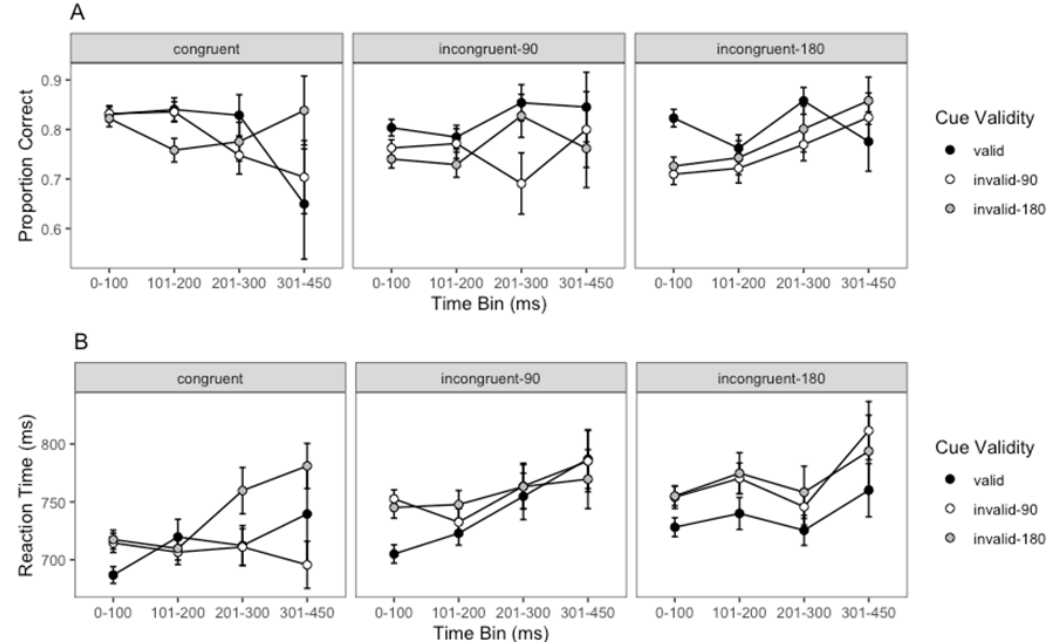

Cue Validity

- valid

invalid-90

○ invalid-180
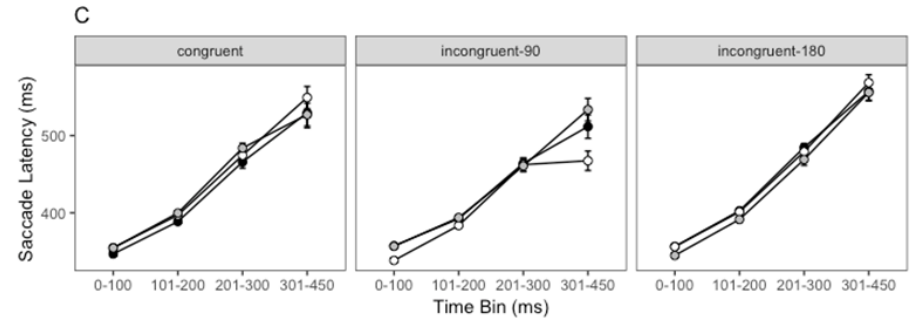

\section{FIGURE A1.}

Proportion correct (Panel A), reaction time (in milliseconds, Panel B), and saccade latency (in milliseconds, Panel C) as a function of saccade congruency, cue validity, and absolute time bin. Error bars represent within-subject SEM. 


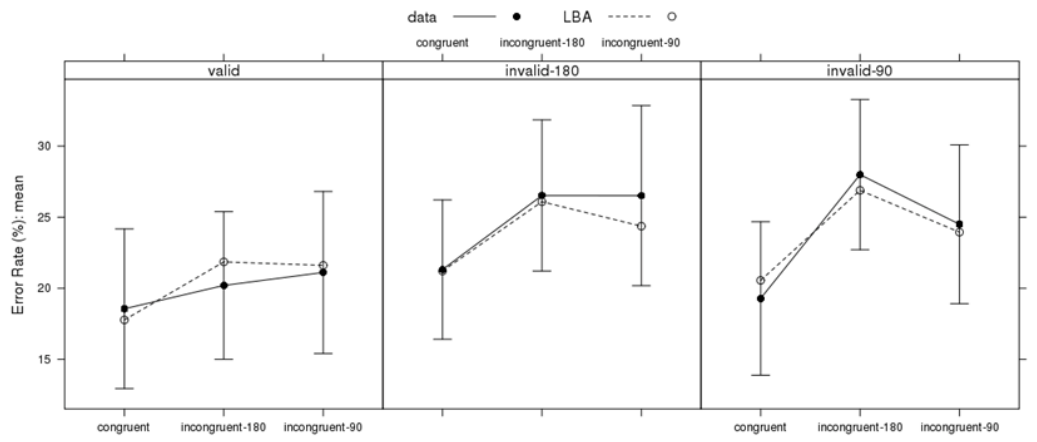

B

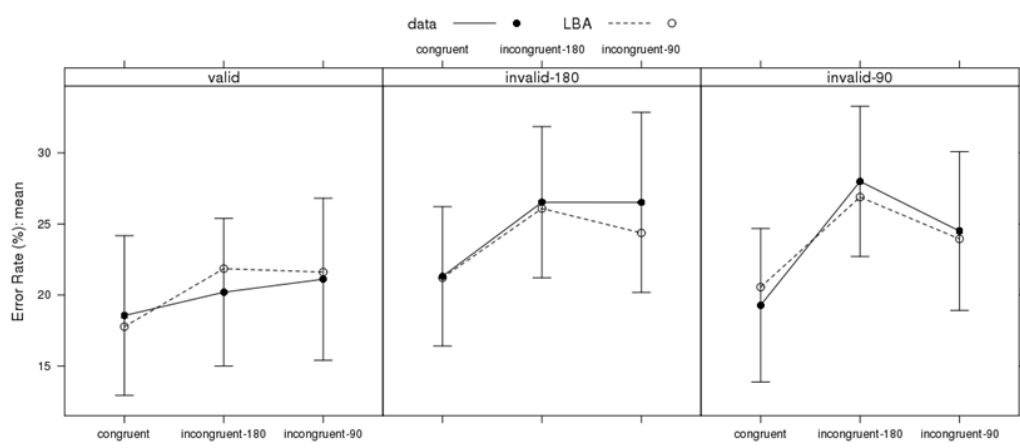

FIGURE A2.

Linear ballistic model fits to error rates (Panel A) and correct RT distribution (Panel B, 10th, 50th and 90th percentiles are lower, middle and upper lines respectively). The abscissa is cue validity and the panels are levels of saccade congruency. Error bars represent 95\% Cls.

\section{Linear Ballistic Accumulator Analysis}

\section{MODEL FIT}

Figure A2 demonstrates that the selected linear ballistic accumulator (LBA) model provides a reasonable account of saccade congruency and cue validity on average accuracy and the distribution of RT. Figure A2, Panel A shows that the model provides a good account of error rate. Figure A2, Panel B represents the distribution of RT in terms of the 10th percentile (representing the fastest responses), 50th percentile (representing the average responses) and the 90th percentile (representing the slowest RT). The account given is quite accurate, with appreciable misfit only for the slowest RTs. This misfit may be the result of our task instructions emphasizing the accuracy of the task, rather speed, causing some rechecking of responses before responding on a minority of trials, a process that is not taken account of by the LBA model.

\section{Linear Ballistic Accumulator Modeling of Saccade Latency}

\section{BAYESIAN SAMPLING METHODS}

For each model, three times as many chains were used as model parameters. Sampling was carried out in two steps. First, sampling was carried out separately for individual participants in order to get reasonable start points for hierarchical sampling. The results of this step were then used as starting points for sampling the full hierarchical sample. During initial burn-in-period, there was a probability of .05 that a crossover step was replaced with a migration step. After burn-in, only crossover steps were used and sampling continued until the proportional scale reduction factor $\left(R^{\prime \prime}\right)$ was less than 1.1 for all parameters, and the multivariate version was less than 1.1 (Brooks \& Gelman, 1998). Hierarchical estimation assumed independent normal population distributions for each model parameter. Population-mean start points were calculated from the mean of the individual-subject posterior medians and population SDs from their standard deviations, with each chain getting a slightly different random perturbation of these values. Hierarchical sampling used probability .05 migration steps at both levels of the hierarchy during burn-in, and only crossover steps thereafter with thinning set at 5 (i.e., only every 5 th sample was kept), with sampling continuing until $R$ " for all parameters at all levels, and the multivariate $R^{\prime \prime}$ values, were all less than 1.1. The final set of chains were also inspected visually to confirm convergence.

\section{PRIORS}

Priors were chosen to have little influence on estimation. Priors were normal distributions that were truncated below zero for $B, A$ and $s_{\mathrm{v}}$ parameters, and truncated at $0.1 \mathrm{~s}$ for the $T_{0}$ parameter (assuming that responses made in less than $0.1 \mathrm{~s}$ are implausible). The t0 parameter was truncated above by $1 \mathrm{~s}$, and no posterior samples ever approached this limit. There were no other truncations, so the $v$ prior was unbounded. The prior mean for $B$ was 1 and for 0.5 for $A$. The $v$ 


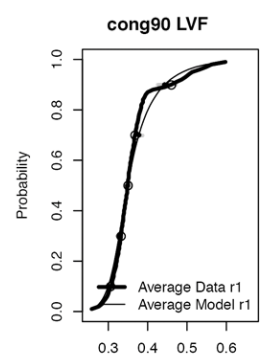

$R T(s)$

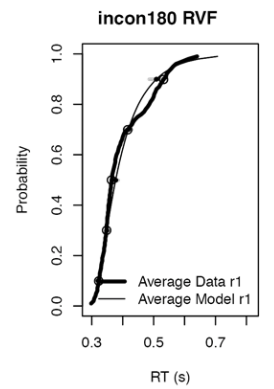

incon180 LVF

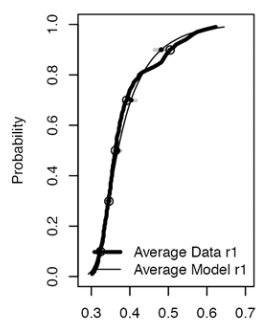

RT (s)

incon90 RVF

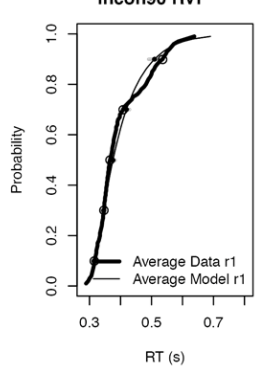

incon90 LVF

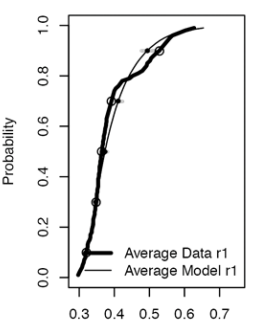

RT (s)
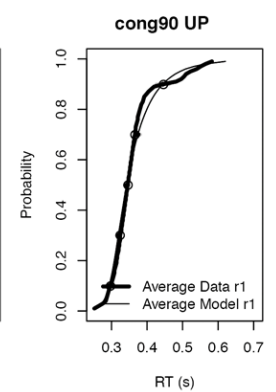

cong90 RVF

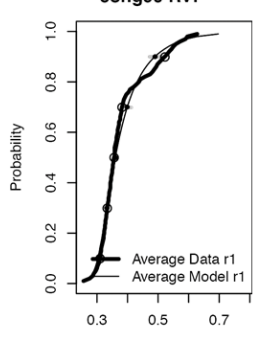

RT (s)

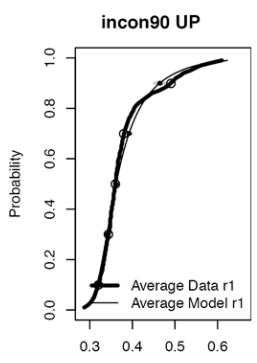

RT (s)

\section{FIGURE A3.}

Cumulative density functions. Starting at top left and moving clockwise, the panels represent trials where (a) the saccade goal and spatial cue coincide at the same left visual field (LVF) placeholder position, (b) the saccade goal is in the LVF and spatial cue appears at a visual angle of $180^{\circ}$ from the saccade goal in the right visual field (RVF), (c) the saccade goal is in the LVF and the spatial cue appears at a visual angle of $90^{\circ}$ from this location (placeholder above fixation), (d) the saccade goal and spatial goal are congruent and both appear in the RVF, (e) saccade goal is in the RVF and spatial cue appears at an angle of $180^{\circ}$ (LVF), (f) saccade goal is in the RVF and the spatial cue appears at an angle of $90^{\circ}$ from it in the placeholder above fixation, (g) saccade goal and spatial cue both appear in the upward placeholder, and (h) the saccade goal is in the upward placeholder and the spatial cue appears either in the LVF or RVF.

parameter was given a prior mean of 1 and the $s_{\mathrm{v}}$ parameter had a prior mean of 0.5 . The $T_{0}$ parameter had a prior mean of $0.3 \mathrm{~s}$. All priors had a standard deviation of 2. Mean parameters of population distributions were assumed to have priors of the same form as for individual estimation, and the SDs of hyper parameters were assumed to have exponential distributions with a scale parameter of 1 . Plots of prior and posterior distributions revealed strong updating (i.e., posteriors dominated priors), making it clear that the prior assumptions had little influence on posterior estimates.

\section{MODEL FIT}

Figure A3 displays the fit of the LBA model to the saccade latency data in terms of defective cumulative distribution functions (lines) and 10th, 30th, 50th, 70th and 90th percentiles (points from left to right) averaged over participants. The thick black line and open points correspond to the data and the thin grey lines solid black points to the model prediction averaged over posterior samples. The grey points correspond to percentile predictions for 100 randomly selected sets of posterior parameter samples, so their spread gives an idea of the uncertainty in the model's predictions. Figure A3 shows that the average fit of the selected LBA model was good.

\section{REFERENCES}

Brooks, S. P., \& Gelman, A. (1998). General methods for monitoring convergence of iterative simulations. Journal of Computational and Graphical Statistics, 7, 434-455. doi: 10.1080/10618600.1998.10474787 الس الس الس 